\title{
O uso de tecnologias digitais no ensino de geometria espacial: uma revisão da
}

\section{literatura}

\author{
The use of digital technologies in spatial geometry teaching: a literature review \\ El uso de tecnologías digitales en la enseñanza de la geometría espacial: una revisión literaria
}

Recebido: 03/11/2021 | Revisado: 10/11/2021 | Aceito: 16/11/2021 | Publicado: 25/11/2021

Francisco César de Oliveira

ORCID: https://orcid.org/0000-0003-1968-4548 Instituto Federal de São Paulo, Brasil

E-mail: francisco.cesar@ifsp.edu.br

Robson Rodrigues da Silva

ORCID: https://orcid.org/0000-0003-1082-0777 Universidade de Mogi das Cruzes, Brasil

E-mail: robson.silva@umc.br

Márcia Aparecida Silva Bissaco

ORCID: https://orcid.org/0000-0002-3219-2567

Universidade de Mogi das Cruzes, Brasil

E-mail:mbissaco@gmail.com

\begin{abstract}
Resumo
$\mathrm{O}$ aprendizado de matemática sempre causou desconforto para muitos estudantes, principalmente durante o Ensino Médio, período de aprofundamento em algumas áreas, como no estudo da Geometria Espacial, tema de complexa abordagem em sala de aula. Objetivo: este estudo tem o propósito de buscar trabalhos na literatura com a finalidade de encontrar evidências quanto ao uso de novas tecnologias digitais no ensino e aprendizagem da Geometria Espacial. Metodologia: para a pesquisa, foram definidas as expressões de busca, as bases de dados e os critérios de inclusão e exclusão. Resultados: após pesquisa nas principais bases de dados nacionais e internacionais, dez artigos foram selecionados para leitura e dois contemplam em parte os objetivos propostos. O primeiro propõe um sistema computacional para a modelagem tridimensional a partir de planos e retas, no entanto, utiliza tecnologias desatualizadas, enquanto o segundo estuda o aprendizado, mas apenas de professores de matemática iniciantes, e não de estudantes. Considerando os mesmos critérios metodológicos anteriores, uma segunda pesquisa foi realizada com a inclusão da expressão "realidade aumentada", resultando em cinco artigos, os quais também são descritos de forma breve em Resultados. Conclusão: os resultados obtidos permitem concluir que, embora a literatura mostre relevantes trabalhos associando o ensino e aprendizagem da Geometria Espacial com tecnologias digitais, a maioria descreve apenas o estudo de sólidos geométricos, onde não se considera outras temáticas dessa área, como posições relativas entre pontos, retas e planos no espaço.
\end{abstract}

Palavras-chave: Aprendizado; Ensino; Geometria espacial; Matemática; Tecnologia.

\begin{abstract}
Learning mathematics has always caused discomfort for many students, especially during high school, a period of deepening in some areas, such as the study of spatial geometry, a complex subject to be addressed in the classroom. Objective: The purpose of this study was to search the literature to find evidences about the use of new digital technologies in teaching and learning spatial geometry. Methodology: for the research, the search terms, the databases, and the inclusion and exclusion criteria were defined. Results: After searching the main national and international databases, ten articles were selected for reading, and two of them partially meet the proposed objectives. The first one proposes a computational system for three-dimensional modeling from planes and lines, however, it uses outdated technologies, while the second one studies learning, but only of beginning mathematics teachers, and not of students. Considering the same previous methodological criteria, a second search was carried out with the inclusion of the expression "augmented reality", resulting in five articles, which are also briefly described in Results. Conclusion: the results obtained allow us to conclude that, although the literature shows relevant works associating the teaching and learning of Spatial Geometry with digital technologies, most of them describe only the study of geometric solids, where other themes of this area are not considered, such as relative positions between points, lines and planes in space.
\end{abstract}

Keywords: Learning; Teaching; Spatial geometry; Math; Technology. 


\begin{abstract}
Resumen
El aprendizaje de las matemáticas siempre ha causado malestar a muchos estudiantes, especialmente durante la escuela secundária, período de profundización en algunas áreas, como es el caso del estudio de la Geometría Espacial, una asignatura de abordaje complejo en el aula. Objetivo: este estudio tiene como objetivo la búsqueda de trabajos en la literatura con el fin de encontrar evidencias sobre el uso de las nuevas tecnologías digitales en la enseñanza y el aprendizaje de la Geometría Espacial. Metodología: para la investigación se definieron expresiones de búsqueda, bases de datos y criterios de inclusión y exclusión. Resultados: luego de buscar en las principales bases de datos nacionales e internacionales, se seleccionaron diez artículos para lectura y dos contemplan parcialmente los objetivos propuestos. El primero propone un sistema computacional para modelado tridimensional a partir de planos y rectas, sin embargo, utiliza tecnologías obsoletas, mientras que el segundo estudia el aprendizaje, pero solo para profesores principiantes de matemáticas, y no para estudiantes. Considerando los mismos criterios metodológicos anteriores, se realizó una segunda búsqueda con la inclusión de la expresión "realidad aumentada", em lo que resultó cinco artículos, que también se describen brevemente en Resultados. Conclusión: los resultados obtenidos permiten concluir que, si bien la literatura muestra trabajos relevantes que asocian la enseñanza y el aprendizaje de la Geometría Espacial con las tecnologías digitales, la mayoría describe únicamente el estudio de los sólidos geométricos, donde otros temas de esta área no son considerados, como posiciones relativas entre puntos, rectas y planos en el espacio.
\end{abstract}

Palabras clave: Aprendizaje; Enseñanza; Geometría espacial; Matemáticas; Tecnología.

\title{
1. Introdução
}

Dentre os currículos disciplinares oferecidos pelas escolas em todo o mundo, a matemática representa um dos mais difíceis para os alunos, em especial durante os primeiros anos da vida escolar. Pesquisadores do assunto, dentre eles Watt (2004) e Frenzel et al. (2010), demonstraram que o interesse dos alunos em matemática diminui consistentemente ao longo da adolescência. Marsh et al. (2005) entendem que o interesse nos primeiros anos escolares é muito importante para o desenvolvimento dos estudantes ao longo de sua vida acadêmica, e dada essa relevância para o processo de aprendizagem bem sucedido, é importante que o professor busque formas de manter o interesse do aluno, como por exemplo, considerar recursos lúdicos, a informática, além do próprio ambiente escolar (Silva et al., 2020).

Para Slezáková (2011), o estudante não possui uma "imaginação geométrica" suficiente para resolver problemas geométricos. Tais problemas, de acordo com Ferdiánová (2017), pode ser fruto de diferentes motivos. Um deles é o pouco cuidado que os educadores prestam ao ensino da geometria, outro é o próprio aluno que se vê desmotivado pela antipatia frente ao assunto. Os alunos possuem habilidades diferentes para desenvolver a imaginação espacial e geométrica, onde o sucesso desse desenvolvimento depende do processo educacional e aprendizado adequados (Slezáková, 2011).

Estudos realizados por Battista (1990) reforçam que as habilidades espaciais apoiam o pensamento e a realização geométrica. Os trabalhos de Maier (1996) e Yilmaz (2009) igualmente reforçam a importância em se ter habilidade espacial para mentalizar e visualizar imagens e objetos, seja com as disciplinas de Física, Matemática e Engenharias. Bennie \& Smit (1999) destacam que sem habilidades espaciais, uma pessoa não seria capaz de identificar os locais, direções, distâncias, tamanho ou formas dos objetos do mundo real, sendo esta altamente relevante para a vida cotidiana do indivíduo.

O pensamento espacial sustenta a vida cotidiana, o trabalho e a ciência de forma poderosa, representando diversos papeis, que envolve a compreensão de metáforas, a interpretação de obras de arte, a modelagem molecular, a realização de provas de geometria, além de outros. Esse tipo de pensamento pode ser apoiado por quaisquer um dos sentidos do homem ou por outras ferramentas, como lápis e papel, assim como outras mais tecnológicas (Downs \& Desouza, 2006). Battista \& Clements (1996) sustentam que a aprendizagem de cálculo de volumes sólidos gera dificuldade porque os estudantes nem sempre conseguem mentalizar o tridimensional a partir do bidimensional, ou seja, converter mentalmente imagens bidimensionais em tridimensionais.

De acordo com Mayer (1987), a teoria do desenvolvimento cognitivo descrita por Piaget formula que o aluno do Ensino Fundamental se encontra na fase concreta e aprende tomando em conta as características físicas dos objetos, como formas, cores e tamanhos, onde a maioria desses alunos possuem apenas a capacidade de resumir, por exemplo, as 
propriedades físicas de uma imagem, mas não conseguem realizar outras comparações abstratas. Van Hiele (1986) e Chang et al. (2007) concluem que quando uma representação não é suficientemente concreta, os alunos tendem a experimentar dificuldades de aprendizagem.

Garrity (1998) e Gurny (2003) descrevem que professores de matemática podem ser incapazes de ajudar o estudante a ter um mecanismo de pensamento eficaz para construir imagens espaciais e manipulá-las enquanto resolvem a geometria tridimensional. Concluem os autores que estudos apontam que cada vez mais a atenção vem sendo direcionada sistematicamente à competência do professor como um dos fatores determinantes para a qualidade do ensino de matemática. Professores e alunos relatam dificuldades em relação ao ensino e aprendizagem de geometria espacial, onde o estudante experimenta algumas dificuldades nesse processo, como imaginar um sólido ou realizar cálculos algébricos vinculados à geometria, o que impede o adequado aprendizado da geometria (Chaquiam et al., 2020).

Um instrumento avaliativo que busca medir o desempenho de estudantes nas áreas de leitura, matemática e ciências é o PISA (Programme for International Student Assessment). O censo de 2018, cujos dados foram publicados em 2020, insere o Brasil na $66^{\mathrm{a}}$ posição na área da matemática, demandando especial atenção.

Diante disso, foi realizado uma revisão sistemática com o objetivo de encontrar evidências de trabalhos relevantes que possam trazer a problemática proposta, a qual relaciona matemática, ensino, aprendizagem, tecnologias digitais, estudantes do Ensino Médio e Geometria Espacial, tema de difícil implementação em sala de aula.

\section{Metodologia}

A metodologia teve o objetivo de evidenciar estudos científicos a nível nacional e internacional relacionados com novas tecnologias em processos de ensino e aprendizagem de Geometria Espacial por estudantes dos Ensinos Fundamental e Médio. Para efeito de cobertura temporal, foram considerados trabalhos publicados entre 1988 a 2021.

Inicialmente foram pesquisados trabalhos relacionados com a matemática, o ensino, o aprendizado e a sala de aula. Considerando que o estudo objetiva associar a Geometria Espacial com novos formatos tecnológicos de aprendizagem, na segunda busca foram adicionados os descritores geometria, espacial e tecnologia. Após leitura em diversos artigos desse grupo, observou-se que os descritores geometria e espacial se mostraram totalmente fora do contexto da revisão, uma vez que se observa desconexão entre essas expressões na maioria dos casos. Por exemplo, nos casos de "geometria molecular" ou "habilidade espacial", onde os descritores geometria e espacial não se relacionam com a matemática no contexto desse trabalho. Com isso, uma terceira e última pesquisa resultou em 42 trabalhos, sendo que 32 foram excluídos por não contemplarem estudos com a temática da Geometria Espacial. Os dez trabalhos restantes são descritos no capítulo Resultados.

A pesquisa foi realizada durante os meses de agosto e setembro de 2021 através do Portal Capes. Para o mecanismo de busca foram utilizadas as expressões "math*" ou "matemática" obrigatórias no título e com a presença de pelo menos uma das seguintes expressões, também no título: "teach", "learning", "ensino", "aprendizagem", "aprendizado", "professor", "classroom" ou "sala de aula". As buscas seguintes incluíram as expressões "geometria", "espacial" e "tecnologia" (e os seus correspondentes no idioma inglês) em quaisquer partes dos textos dos trabalhos. Os critérios de inclusão e exclusão são descritos no Quadro 1. 
Quadro 1. Critérios de inclusão e exclusão utilizados na pesquisa.

\begin{tabular}{|c|c|}
\hline Inclusão & Exclusão \\
\hline $\begin{array}{l}\text { Apenas estudos relacionados com o ensino/aprendizagem de Matemática } \\
\text { mediados por tecnologias digitais }\end{array}$ & Trabalhos duplicados \\
\hline $\begin{array}{l}\text { Abrangência das palavras "matemática", "ensino”, "aprendizagem”, } \\
\text { "aprendizado", "professor", "sala de aula", "geometria espacial" e } \\
\text { "tecnologia" nos sistemas de buscas; e o mesmo para expressões em inglês }\end{array}$ & $\begin{array}{l}\text { Estudos onde as palavras } \\
\text { "espacial", "geometria", "spatial" } \\
\text { e "geometry" não se relacionem } \\
\text { com Geometria Espacial }\end{array}$ \\
\hline $\begin{array}{l}\text { Todas a variações das palavras "math" e "teach", sendo elas "math", } \\
\text { "mathematic", "mathematics", "mathematical", "teach", "teacher" e } \\
\text { "teaching”" }\end{array}$ & $\begin{array}{l}\text { Trabalhos com menos de quatro } \\
\text { páginas (artigos não completos) }\end{array}$ \\
\hline
\end{tabular}

Fonte: Autores (2021).

Conforme Quadro 1, os critérios de inclusão definem estudos relacionando o ensino e/ou aprendizado de matemática mediados por tecnologias digitais, a abrangência de descritores predefinidos, além de todas as variações possíveis de alguns termos em inglês. Como critérios de exclusão, foi considerado estudos duplicados, trabalhos onde as palavras "espacial” e "geometria" não se enquadram no contexto da matemática, e os trabalhos com menos de quatro páginas, considerados não completos.

\section{Resultados e Discussão}

A metodologia empregada na seleção dos estudos abrangeu três pesquisas. A primeira, e com expressões apenas nos títulos dos trabalhos, resultou em mais de 36 mil estudos. Nas duas pesquisas seguintes foram incluídas as expressões "espacial", "geometria" e "tecnologia" em todas as partes dos textos, com a diferença que na terceira e última, as palavras “espacial” e "geometria” foram unidas em uma única expressão afim de evitar estudos não relacionados com os objetivos dessa revisão. O mesmo procedimento foi realizado com expressões em inglês e os resultados das três pesquisas são ilustrados no fluxograma da Figura 1.

Figura 1. Método de busca conforme descritores e critérios utilizados na pesquisa.

\begin{tabular}{|c|c|c|c|c|c|}
\hline \multicolumn{6}{|c|}{$\begin{array}{l}\text { Pesquisa 1: Título: (math* OR matemat*) AND (teach* OR learning OR classroom OR ensino OR } \\
\text { professor OR aprendizagem OR aprendizado OR "sala de aula") } \\
\text { (36.611 trabalhos) }\end{array}$} \\
\hline \multicolumn{6}{|c|}{$\begin{array}{l}\text { Pesquisa 2: Titulo: (math* OR matemat*) AND (teach* OR learning OR classroom OR ensino OR } \\
\text { professor OR aprendizagem OR aprendizado OR "sala de aula") } \\
\qquad \text { Qualquer parte: (espacial AND geometria AND tecnologia) OR (spatial AND geometry AND } \\
\text { technology) (478 trabalhos) }\end{array}$} \\
\hline $\begin{array}{l}\text { Scopus } \\
(232)\end{array}$ & $\begin{array}{l}\text { Science Direct } \\
\text { (15) }\end{array}$ & $\begin{array}{l}\text { ProQuest } \\
\text { (23) }\end{array}$ & $\begin{array}{l}\text { Web of Science } \\
\text { (18) }\end{array}$ & & $\begin{array}{l}\text { Outras Bases } \\
(190)\end{array}$ \\
\hline \multicolumn{3}{|c|}{$\begin{array}{l}\text { Pesquisa 3: } \\
\text { Titulo: (math* OR matemat") AND (teach * OR learning OR } \\
\text { classroom OR ensino OR professor OR aprendizagem OR } \\
\text { aprendizado OR "sala de aula") } \\
\text { Qualquer parte: ("geometria espacial" AND tecnologia) OR } \\
\text { ("spatial geometry" AND technology) } \\
\text { (42 artigos) }\end{array}$} & \multicolumn{2}{|c|}{$\begin{array}{l}\text { Excluidos por } \\
\text { nåo contemplar } \\
\text { o estudo da } \\
\text { Geometria } \\
\text { Espacial: } \\
32 \text { artigos }\end{array}$} & $\begin{array}{l}\text { Separados } \\
\text { para } \\
\text { leitura: } \\
10 \text { artigos }\end{array}$ \\
\hline
\end{tabular}

Fonte: Autores (2021).

Como se observa na Figura 1, a metodologia resultou em três pesquisas consecutivas. Em todas, os descritores utilizados nos títulos dos trabalhos foram sempre os mesmos, onde foi definido a exigência do descritor matemática, além da presença obrigatória de pelos menos um dos seguintes termos: ensino, aprendizagem, professor ou sala de aula. Na segunda 
pesquisa foram incluídos os descritores geometria, espacial e tecnologia, no entanto, a maioria dos 478 trabalhos observados não mostraram relação com a Geometria Espacial. Com isso, na terceira e última pesquisa, dois desses descritores foram incluídos em uma única expressão ("geometria espacial"), reduzindo os trabalhos para 42. Desses, ainda foi preciso excluir outros 32 trabalhos porque os estudos não estão relacionados com processos de ensino e/ou aprendizagem de Geometria Espacial mediados por tecnologias digitais. Ressalta-se que foram consideradas todas as variações desses descritores nos idiomas português e inglês, conforme descrevem o Quadro 1 e a Figura 1. Os dez artigos selecionados para leitura são listados em ordem cronológica no Quadro 2 e no subcapítulo seguinte são descritos os resultados de cada estudo.

Quadro 2. Artigos selecionados para leitura.

\begin{tabular}{|c|c|c|c|c|c|}
\hline \multicolumn{2}{|c|}{ Artigo/Ano } & \multirow{2}{*}{$\begin{array}{c}\text { Título } \\
\begin{array}{l}\text { Using computers to enhance the } \\
\text { mathematics classroom }\end{array}\end{array}$} & \multirow{2}{*}{$\begin{array}{c}\text { Autores } \\
\text { Ayers \& Bitter }\end{array}$} & \multirow{2}{*}{$\begin{array}{c}\text { Temática } \\
\text { Uso de computadores no } \\
\text { ensino de matemática }\end{array}$} & \multirow{2}{*}{$\begin{array}{c}\text { Revista } \\
\begin{array}{c}\text { ACM SIGCUE } \\
\text { Outlook }\end{array}\end{array}$} \\
\hline 1 & 1988 & & & & \\
\hline 2 & 1996 & Computer-Based Mathematics Learning & McCoy & $\begin{array}{l}\text { Linguagens e sistemas } \\
\text { computacionais; } \\
\text { aprendizado de } \\
\text { matemática }\end{array}$ & $\begin{array}{l}\text { Journal of } \\
\text { Research on } \\
\text { Computing in } \\
\text { Education }\end{array}$ \\
\hline 3 & 2002 & $\begin{array}{l}\text { MAT }^{3 \mathrm{D}} \text { : A Virtual Reality Modeling } \\
\text { Language Environment for the Teaching } \\
\text { and Learning of Mathematics }\end{array}$ & $\begin{array}{l}\text { Pasqualotti \& } \\
\text { Freitas }\end{array}$ & $\begin{array}{l}\text { Ensino e aprendizado de } \\
\text { matemática }\end{array}$ & $\begin{array}{l}\text { CyberPsycholog } \\
\text { y \& Behavior }\end{array}$ \\
\hline 4 & 2010 & $\begin{array}{l}\text { Analysis of the revisions that pre-service } \\
\text { teachers of mathematics make of their } \\
\text { own project included in their learning } \\
\text { portfolio }\end{array}$ & Cáceres et al. & Formação de professores & $\begin{array}{l}\text { Teaching and } \\
\text { Teacher } \\
\text { Education }\end{array}$ \\
\hline 5 & 2010 & $\begin{array}{c}\text { Engaging in problem posing activities in a } \\
\text { dynamic geometry settingand the } \\
\text { development of prospective teachers' } \\
\text { mathematical }\end{array}$ & Lavy \& Shriki & Formação de professores & $\begin{array}{l}\text { The Journal of } \\
\text { Mathematical } \\
\text { Behavior }\end{array}$ \\
\hline 6 & 2011 & $\begin{array}{l}\text { A comparative study of the effects of } \\
\text { using dynamic geometry software and } \\
\text { physical manipulatives on the spatial } \\
\text { visualisation skills of pre-service } \\
\text { mathematics teachers }\end{array}$ & Baki et al. & $\begin{array}{c}\text { Tecnologias } \\
\text { computacionais e } \\
\text { habilidades matemáticas }\end{array}$ & $\begin{array}{l}\text { British Journal of } \\
\text { Educational } \\
\text { Technology }\end{array}$ \\
\hline 7 & 2012 & $\begin{array}{l}\text { Humans-with-media and continuing } \\
\text { education for mathematics teachers in } \\
\text { online environments }\end{array}$ & Borba & $\begin{array}{l}\text { Tecnologias } \\
\text { computacionais para o } \\
\text { ensino de matemática }\end{array}$ & $\begin{array}{l}\text { ZDM } \\
\text { Mathematics } \\
\text { Education }\end{array}$ \\
\hline 8 & 2013 & $\begin{array}{l}\text { Sense-making and structure in teachers' } \\
\text { reception of educational reform. A case } \\
\text { study on statistics in the mathematics } \\
\text { curriculum }\end{array}$ & $\begin{array}{c}\text { März \& } \\
\text { Kelchtermans }\end{array}$ & Políticas educacionais & $\begin{array}{l}\text { Teaching and } \\
\text { Teacher } \\
\text { Education }\end{array}$ \\
\hline 9 & 2016 & $\begin{array}{c}\text { Effects of Teaching Activities via Google } \\
\text { Sketchup and Concrete Models on Spatial } \\
\text { Skills of Preservice Mathematics } \\
\text { Teachers }\end{array}$ & Uygan \& Kurtuluş & $\begin{array}{l}\text { Ferramentas web para } \\
\text { modelagem 3D }\end{array}$ & $\begin{array}{l}\text { Turkish Journal } \\
\text { of Computer and } \\
\text { Mathematics } \\
\text { Education }\end{array}$ \\
\hline 10 & 2017 & $\begin{array}{l}\text { Design approach of mathematics learning } \\
\text { activities in a digital environment for } \\
\text { children with autism spectrum disorders }\end{array}$ & Santos et al. & $\begin{array}{c}\text { Aprendizado de } \\
\text { matemática de crianças } \\
\text { autistas }\end{array}$ & $\begin{array}{l}\text { Education Tech } \\
\text { Research dev }\end{array}$ \\
\hline
\end{tabular}

Fonte: Autores (2021).

\subsection{Análise dos Resultados}

Os oito primeiros artigos descritos a seguir apresentam pouca ou nenhuma relação com os interesses dessa revisão, enquanto os dois últimos, uma proximidade maior. Ayers e Bitter (1988) fazem um estudo sobre o comportamento do 
professor de matemática frente ao ensino mediado por computador. Apesar de relacionar tendências tecnológicas e ensino de matemática, foi escrito há mais de trinta anos, além de estar centrado apenas no professor.

O trabalho proposto por McCoy (1996) descreve linguagens de programação e alguns sistemas que foram utilizados entre o final da década de 1980 e o início da seguinte. Embora trate exclusivamente de tecnologias computacionais para o aprendizado de matemática no formato de revisão da literatura, não faz menção à Geometria Espacial e não aborda procedimentos e tecnologias atuais.

O artigo 4 estuda alunos universitários e como eles, futuros professores de matemática, aprenderam os diferentes conteúdos pertinentes. Escrito por Cáceres et al. (2010), a pesquisa não se relaciona com tecnologias computacionais para o ensino e/ou aprendizado de matemática.

Assim como o anterior, o trabalho descrito por Lavy e Shriki (2010) estuda a formação de professores de matemática, e como eles aprenderam os conceitos relacionados, dentre eles, a temática da geometria. Não está relacionado com tecnologias computacionais para o ensino e/ou aprendizagem de matemática.

A proposta de Borba (2012) considera o ano de 2012 para averiguar os recursos tecnológicos utilizados durante os anos anteriores no Brasil. Embora concentre os estudos no ensino da matemática, não apresenta nenhuma relação com a Geometria Espacial.

O artigo descrito por März e Kelchtermans (2013) aborda políticas públicas voltadas para a administração do ensino secundário na Bélgica e não se relaciona com soluções computacionais para o ensino, apenas mostra um estudo exploratório baseado em entrevistas com vinte amostras experimentais com professores do Ensino Médio.

Outro estudo experimental é mostrado por Uygan e Kurtuluş (2016). A pesquisa estuda o desenvolvimento de modelagens 3D através do Google SketchUp por professores de matemática iniciantes na carreira. O experimento considera 72 professores do Ensino Fundamental do ano acadêmico de 2009-2010 de uma Universidade da Turquia, e assim como o anterior, é direcionado para o professor, e não para o aluno.

O estudo apresentado por Santos et al. (2017) relaciona e discute as dificuldades no aprendizado de matemática por crianças autistas entre seis e doze anos de idade. Não apresenta uma relação direta com Geometria Espacial, apenas contextualiza o ensino e o aprendizado de questões como rotação e translação de imagens 2D em base ao protótipo computacional proposto e denominado LEMA (Learning Environment Mathematics Autistic).

A seguir são descritos os trabalhos com mais afinidade com essa revisão. Pasqualotti e Freitas (2002) buscam evidências se novas tecnologias podem auxiliar o aprendizado de Geometria Espacial em processos de ensino. O estudo propõe o MAT3D para a modelagem de figuras geométricas a partir de planos e retas. Os autores apresentam um estudo onde os participantes utilizam um ambiente virtual modelado em VRML ${ }^{1}$. Dos 42 alunos da $7^{\text {a }}$ série selecionados para o estudo, 22 (grupo experimental) conseguiram assimilar por mais tempo o aprendizado adquirido.

A ferramenta MAT3D está estruturada em três componentes: ambiente conhecido, representações matemáticas e avaliação do desempenho dos estudantes. No "ambiente conhecido", o usuário tem acesso a um cenário tridimensional baseado em uma cidade, com suas ruas, avenidas, prédios e demais. No componente "representações matemáticas", o aluno tem como associar as formas do cenário, como planos das paredes dos edifícios e os fios de eletricidade dos postes, com planos e retas, respectivamente. Essas associações se baseiam em transformações dos cenários a medida que o usuário vai criando o seu conhecimento relacionado às formas tridimensionais que o programa permite construir. O objetivo de implementar o ambiente virtual dessa maneira é que ele permite aos alunos construir seu próprio conhecimento em relação ao conteúdo matemático através da simples manipulação de objetos tridimensionais inseridos no ambiente. O terceiro componente mede a avaliação do desempenho dos estudantes, contabilizando as suas respostas através de um formulário eletrônico disponível no ambiente

${ }^{1}$ VRML (Virtual Reality Modeling Language): uma das linguagens de programação utilizadas para o desenvolvimento de cenários virtuais. 
virtual. Durante o processo, o aluno recebe feedback de seu desempenho e, em base aos erros e acertos, pode retornar com a possibilidade de refazer o procedimento, fazendo do ambiente virtual um processo dinâmico, conforme descrevem os autores.

Outro trabalho relacionado é descrito por Baki et al. (2011). O estudo compara as habilidades de visualização espacial de professores de matemática do primeiro ano do ensino fundamental mediante dois métodos com o grupo experimental, sendo o primeiro mediante um software de geometria dinâmica e o segundo através de manipuladores físicos, enquanto o método tradicional de ensino é utilizado com o grupo controle (terceiro método).

O primeiro método compreende a utilização do software Cabri 3D. O sistema permite explorar a geometria 3D através de visualizações em um ambiente computacional onde o aluno pode explorar relações geométricas entre objetos planos e tridimensionais, além de fazer e testar conjeturas entre eles. Uma característica distinta, conforme os autores, é a capacidade de construir objetos geométricos e especificar relacionamentos entre eles quando se utiliza o software Cabri 3D.

Os manipuladores físicos, correspondente ao segundo método, são frequentemente utilizados em salas de aula para ajudar os alunos a compreenderem conceitos abstratos, principalmente sobre volumes e áreas de superfícies tridimensionais, e geralmente são utilizados por professores, podendo facilitar o ensino e a aprendizagem de matemática dos alunos. O terceiro e último método utilizado é o tradicional, os quais são baseados apenas em imagens bidimensionais, geralmente através de lousa ou ilustrações de livros.

Os autores utilizaram a metodologia PSVT (Purdue Spatial Visualization Test) para estudar 96 professores de matemática iniciantes na profissão, sendo 66 do grupo controle e o restante do grupo experimental. A metodologia PSVT foi desenvolvida por Roland Guay em 1976, sendo baseada em três seções de testes. A seção desenvolvimentos exige que os alunos estudem um padrão de objetos 3D e determinem a resposta correta entre as cinco formas possíveis. Por exemplo, é mostrado uma imagem que representa uma forma 3D "desmontada" no plano, e como possíveis respostas são apresentadas cinco opções. Na seção rotações cada pergunta mostra o mesmo objeto 3D em duas posições diferentes, onde a segunda posição é resultado de uma rotação da primeira em quaisquer um dos três eixos. Um segundo objeto 3D em uma única posição é mostrado ao aluno e este deve marcar a resposta correta de acordo ao padrão de rotação mostrado com o primeiro objeto. Por fim, a seção visualizações testa a capacidade do aluno de visualizar um objeto 3D sob várias perspectivas.

Os resultados demonstraram que as pontuações do teste PSVT dos grupos que utilizaram os métodos baseados tanto no software Cabri 3D, quanto nos manipuladores físicos, ambos do grupo experimental, obtiveram melhores resultados de aprendizado quando comparados com o método tradicional de ensino (grupo controle). Demonstrou-se, ainda, que o método através do software permitiu aos usuários construírem suas próprias figuras geométricas, aprimorando as suas habilidades de visualização espacial.

Uma tecnologia que ganha entusiastas a cada ano é a realidade aumentada, encontrada em praticamente todas as áreas do conhecimento, inclusive na Educação. A realidade aumentada é definida por Bajura e Neumann (1995) como uma técnica capaz de sobrepor objetos virtuais no mundo real mediante a utilização de algum recurso tecnológico, incrementando, dessa forma, a visão do usuário. Krebs et al. (2019) apresentou um estudo onde a realidade aumentada aplicada em celulares se mostrou como um instrumento auxiliador em processos de ensino e aprendizagem, o que pode motivar os alunos dentro da sala de aula. Do ponto de vista pedagógico, a técnica de realidade aumentada pode ser muito útil para o aprendizado do aluno do Ensino Fundamental, criando expectativas de aprendizagem em sala de aula (Hidayat et al., 2021).

Utilizando a mesma plataforma de busca e com os mesmos descritores do primeiro estudo, acrescido da expressão "realidade aumenta" nos idiomas português e inglês no título, e com a única diferença que foi considerado os últimos seis anos (de 2016 a 2021), uma segunda pesquisa obteve cinco trabalhos mais recentes, os quais são listados no Quadro 3 e descritos de forma breve a seguir. 
Quadro 3. Pesquisa com os mesmos critérios do primeiro estudo, acrescido dos descritores "realidade aumentada" ou “augmented reality" no título dos artigos, e considerando apenas os últimos seis anos de publicação.

\begin{tabular}{|c|c|c|c|}
\hline Autores/Ano & Título & Objetivo & Revista \\
\hline $\begin{array}{c}\text { De Cassio Macedo et } \\
\text { al. (2016) }\end{array}$ & $\begin{array}{c}\text { Usando Smartphone e Realidade } \\
\text { Aumentada para Estudar Geometria } \\
\text { Espacial }\end{array}$ & $\begin{array}{c}\text { Mostrar um aplicativo para a } \\
\text { visualização de pirâmides por } \\
\text { estudantes do Ensino Médio }\end{array}$ & RENOTE \\
\hline Gomes et al. (2019) & $\begin{array}{c}\text { GeometriAR: aplicativo educacional com } \\
\text { realidade aumentada para auxiliar o } \\
\text { ensino de sólidos geométricos }\end{array}$ & $\begin{array}{c}\text { Estudar a visualização de figuras } \\
\text { sólidas com nove professores } \\
\text { especialistas }\end{array}$ & RENOTE \\
\hline Lee (2019) & $\begin{array}{c}\text { Using augmented reality to train students } \\
\text { to visualize three-dimensional drawings } \\
\text { of mortise-tenon joints in furniture } \\
\text { carpentry }\end{array}$ & $\begin{array}{c}\text { Visualização 3D por estudantes } \\
\text { de carpintaria para facilitar o } \\
\text { processo de produção de móveis }\end{array}$ & $\begin{array}{c}\text { Interactive } \\
\text { Environments }\end{array}$ \\
\hline $\begin{array}{c}\text { Ibili et al. (2019) } \\
\text { Eerceptions towards an augmented reality } \\
\text { tutoring system }\end{array}$ & $\begin{array}{c}\text { Applying the technology acceptance } \\
\text { model to understand maths teachers' } \\
\text { realidade aumentada para } \\
\text { Geometria Espacial }\end{array}$ & $\begin{array}{c}\text { Education and } \\
\text { Technologies }\end{array}$ \\
\hline $\begin{array}{c}\text { Fernández \& Delgado } \\
\text { (2020) }\end{array}$ & $\begin{array}{c}\text { Augmented Reality as a Didactic } \\
\text { Resource for Teaching Mathematics }\end{array}$ & $\begin{array}{c}\text { Propor um aplicativo para a } \\
\text { modelagem de poliedros }\end{array}$ & Applied Sciences \\
\hline
\end{tabular}

Fonte: Autores (2021).

O primeiro (De Cassio Macedo et al., 2016) propõe um estudo de visualizações tridimensionais de pirâmides com alunos de uma turma do Ensino Médio, onde os cenários ocorrem através de um aplicativo desenvolvido para dispositivos móveis. O segundo artigo, proposto por Gomes et al. (2019), apresenta algo semelhante, mas para a visualização virtual de figuras sólidas a partir de marcadores ${ }^{2}$ de realidade aumentada. $\mathrm{O}$ estudo faz uma abordagem de usabilidade e eficiência do aplicativo com nove professores especialistas. Lee (2019) apresenta o terceiro artigo, onde mostra um aplicativo para a visualização e aprendizado de figuras 3D por estudantes técnicos em carpintaria, e tem por objetivo a prática de interações tridimensionais em sala de aula para facilitar a fabricação de móveis. O quarto artigo, de Ibili et al. (2019), apresenta um sistema computacional e faz um estudo com 148 professores de Matemática para saber se a ferramenta tem utilidade no aprendizado de Geometria Espacial. O quinto e último artigo propõe um sistema para a visualização e estudo de poliedros (Fernández \& Delgado, 2020).

\section{Considerações Finais}

Foi revisado na parte introdutória que a matemática sempre causou (e causa) muitos desconfortos em processos de ensino e aprendizagem por parte de professores e alunos. Quando se considera o tema da Geometria Espacial nesses processos, a situação que se observa é ainda mais delicada, pois, como visto, o ensino/aprendizagem dessa temática causa enormes dificuldades por parte dos envolvidos.

Diversos autores relataram a importância em se ter habilidades espaciais, as quais norteiam a nossa vida em muitas de nossas atividades cotidianas, como o ato de caminhar pelas ruas, a conclusão de outras disciplinas relacionadas, como Física, Química e outras, assim como a simples apreciação de uma obra de arte. Como se observa, a Geometria Espacial está presente em inúmeras atividades de nosso dia a dia, repercutindo de forma importante em muitas de nossas ações, sejam elas educacionais ou não.

\footnotetext{
${ }^{2}$ Marcadores de realidade aumentada: espécie de imagem estampada em uma superfície qualquer, como papéis e outros materiais. Quando capturada por uma câmera, a imagem tem a função de se relacionar com algum objeto ou cenário virtual, o qual é projetado em uma tela para o usuário.
} 
O conhecimento adequado da Geometria Espacial depende fortemente de boas práticas acadêmicas, o que nem sempre é logrado com êxito, seja pelo professor que dedica pouco cuidado ao tema, ou mesmo pelo aluno que enxerga a matemática com certa antipatia natural. Isso fica mais evidente quando se observa o último PISA, onde o desempenho do estudante brasileiro na área de matemática demanda especial atenção, uma vez que ocupamos a $66^{\mathrm{a}}$ posição no ranking global.

Por outro lado, soluções digitais têm surgido com o propósito de facilitar processos educacionais, inclusive no aprendizado de Geometria Espacial, e diante disso, esse estudo selecionou dez artigos para leitura e dois apresentaram alguma relação com os interesses dessa revisão. O primeiro utiliza dispositivos virtuais de visualização pouco utilizados atualmente, como capacetes e luvas 3D (Pasqualotti \& Freitas, 2002), enquanto o segundo (Baki et al., 2011) tem como público alvo o professor de matemática iniciante na carreira, e não o estudante. Os demais estudos, embora tenham sido incluídos conforme critérios definidos, não mostram interesse, já que abordam formação de professores, políticas educacionais, estudantes autistas ou, na maioria dos casos, referem-se a estudos antigos, não tendo, portanto, abordagem relevante com novas tecnologias digitais.

Sobre a pesquisa realizada com a inclusão das expressões "realidade aumentada" e "augmented reality", destaca-se que esses trabalhos, embora de muita relevância, estão voltados basicamente para a visualização e manipulação de sólidos geométricos, não tendo relação com outros temas da Geometria Espacial do Ensino Médio, como posições relativas entre pontos, retas e planos no espaço, paralelismo e perpendicularidade entre retas e planos, projeções ortogonais e distâncias no espaço entre esses elementos, além do estudo de ângulos e suas relações entre retas e planos.

Conclui-se, por fim, que são raros os trabalhos relacionando: 1) ensino e aprendizagem de Geometria Espacial mediados por novas tecnologias digitais; 2) outros temas da Geometria Espacial que não apenas o estudo dos sólidos geométricos e 3) a questão da acessibilidade em dispositivos computacionais cada vez mais presente, principalmente em dispositivos móveis, outra tendência marcante em mecanismos de ensino e aprendizagem.

Diante desse cenário, estamos desenvolvendo um aplicativo direcionado para smartphones e tablets, com o qual se pretende aplicar algumas atividades de Geometria Espacial não encontradas nos trabalhos revisados, como alguns temas da Geometria de Posição, sendo eles: as representações geométricas entre pontos, retas e planos no espaço, as posições relativas entre esses elementos, assim como as propriedades de paralelismo e perpendicularidade. O aplicativo, de nome SpaceGeo_AR, apresentará funções voltadas tanto para o professor, quanto para o aluno, sendo o destaque a implementação de realidade aumentada, onde será possível visualizar cenários tridimensionais envolvendo os elementos temáticos mencionados. O aplicativo ainda terá recursos de tradução do LIBRAS (Língua Brasileira de Sinais) de forma simultânea para os portadores de deficiência auditiva, outra tendência cada vez mais presente em soluções digitais, pois permite a acessibilidade a esse grupo de pessoas.

\section{Agradecimentos}

Os autores agradecem ao Instituto Federal de São Paulo (IFSP) pela viabilidade e apoio financeiro para a publicação desse estudo.

\section{Referências}

Ayers, S. W., \& Bitter, G. (1988). Using computers to enhance the mathematics classroom. ACM SIGCUE Outlook, 20(1), 115-130. https://doi.org/10.1145/382236.382864

Bajura, M., \& Neumann, U. (1995). Dynamic registration correction in video-based augmented reality systems. IEEE Computer Graphics and Applications, 15(5), 52-60. https://doi.org/10.1109/38.403828 
Baki, A., Kosa, T., \& Guven, B. (2011). A comparative study of the effects of using dynamic geometry software and physical manipulatives on the spatial visualisation skills of pre-service mathematics teachers. British Journal of Educational Technology, 42(2), 291-310. https://doi.org/10.1111/j.14678535.2009.01012.x

Battista, M. T. (1990). Spatial visualization and gender differences in high school geometry. Journal for research in mathematics education, 21(1), 47-60. https://doi.org/10.5951/jresematheduc.21.1.0047

Battista, M. T., \& Clements, D. H. (1996). Students' understanding of three-dimensional rectangular arrays of cubes. Journal for Research in Mathematics Education, 27(3), 258-292. https://doi.org/10.5951/jresematheduc.27.3.0258

Bennie, K., \& Smit, S. (1999). Spatial sense: Translating curriculum innovation into classroom practice. In 5th Annual Congress of the Association for Mathematics Education of South Africa (AMESA), Port Elizabeth. http://academic.sun.ac.za/mathed/malati/files/geometry992.pdf

Borba, M. C. (2012). Humans-with-media and continuing education for mathematics teachers in online environments. ZDM, 44(6), 801-814. https://doi.org/10.1007/s11858-012-0436-8

Cáceres, M. J., Chamoso, J. M., \& Azcárate, P. (2010). Analysis of the revisions that pre-service teachers of Mathematics make of their own project included in their learning portfolio. Teaching and Teacher Education, 26(5), 1186-1195. https://doi.org/10.1016/j.tate.2010.01.003

Chang, K. E., Sung, Y. T., \& Lin, S. Y. (2007). Developing geometry thinking through multimedia learning activities. Computers in Human Behavior, 23(5), 2212-2229. https://doi.org/10.1016/j.chb.2006.03.007

Chaquiam, M., Maués, D. D. D. N., Cabral, N. F., Dias, G. N., Rodrigues, A. E., \& Pamplona, V. M. S. (2020). A percepção de alunos e professores sobre o ensino e aprendizagem do cilindro circular reto. Research, Society and Development, 9(9), e973998110. http://dx.doi.org/10.33448/rsd-v9i9.8110

De Cassio Macedo, A., da Silva, J. A., \& Buriol, T. M. (2016). Usando Smartphone e Realidade aumentada para estudar Geometria espacial. RENOTE, 14(2). https://doi.org/10.22456/1679-1916.70688

Downs, R., \& Desouza, A. (2006). Learning to think spatially: GIS as a support system in the K-12 curriculum. Committee on the Support for the Thinking Spatially, National Research Council, Publisher: The National Academies Press. https://doi.org/10.17226/11019

Ferdiánová, V. (2017). GeoGebra Materials for LMS Moodle Focused Monge on Projection. Electronic Journal of e-Learning, 15(3), 259-268. https://files.eric.ed.gov/fulltext/EJ1146049.pdf

Fernández-Enríquez, R., \& Delgado-Martín, L. (2020). Augmented reality as a didactic resource for teaching mathematics. Applied Sciences, 10(7), 2560. https://doi.org/10.3390/app10072560

Frenzel, A. C., Goetz, T., Pekrun, R., \& Watt, H. M. (2010). Development of mathematics interest in adolescence: Influences of gender, family, and school context. Journal of Research on Adolescence, 20(2), 507-537. https://doi.org/10.1111/j.1532-7795.2010.00645.x

Garrity, C. (1998). Does the Use of Hands-On Learning, with Manipulatives, Improve the Test Scores of Secondary Education Geometry Students?. https://files.eric.ed.gov/fulltext/ED422179.pdf

Gomes, A. P. L., Ramos, R. A., de Brito, L. F., Batista, M. F., \& Leal, B. G. (2019). GeometriAR: aplicativo educacional com realidade aumentada para auxiliar o ensino de sólidos geométricos. RENOTE, 17(1), 405-414. https://doi.org/10.22456/1679-1916.95848

Gurny, H. G. (2003). High School Students' Performance on Vandenberg's Mental Rotations Test: Art Ability, Gender, Activities, Academic Performance, Strategies, and Ease of Taking the Test. https://files.eric.ed.gov/fulltext/ED479372.pdf

Hidayat, H., Sukmawarti, S., \& Suwanto, S. (2021). The application of augmented reality in elementary school education. Research, Society and Development, 10(3), e14910312823. http://dx.doi.org/10.33448/rsd-v10i3.12823

Ibili, E., Resnyansky, D., \& Billinghurst, M. (2019). Applying the technology acceptance model to understand maths teachers' perceptions towards an augmented reality tutoring system. Education and Information Technologies, 24(5), 2653-2675. https://doi.org/10.1007/s10639-019-09925-z

Krebs, D., da Rosa Zucolo, M. P., \& Ghisleni, T. S. (2019). O uso da realidade aumentada aplicado em ensino. Research, Society and Development, 8(7), e1871080. http://dx.doi.org/10.33448/rsd-v8i7.1080

Lavy, I., \& Shriki, A. (2010). Engaging in problem posing activities in a dynamic geometry setting and the development of prospective teachers' mathematical knowledge. The Journal of Mathematical Behavior, 29(1), 11-24. https://doi.org/10.1016/j.jmathb.2009.12.002

Lee, I. J. (2020). Using augmented reality to train students to visualize three-dimensional drawings of mortise-tenon joints in furniture carpentry. Interactive Learning Environments, 28(7), 930-944. https://doi.org/10.1080/10494820.2019.1572629

Maier, P. H. (1996). Spatial geometry and spatial ability-How to make solid geometry solid. In Selected papers from the Annual Conference of Didactics of Mathematics (pp. 63-75). http://webdoc.sub.gwdg.de/ebook/e/gdm/1996/maier.pdf

Marsh, H. W., Trautwein, U., Lüdtke, O., Köller, O., \& Baumert, J. (2005). Academic self-concept, interest, grades, and standardized test scores: Reciprocal effects models of causal ordering. Child development, 76(2), 397-416. https://doi.org/10.1111/j.1467-8624.2005.00853.x

März, V., \& Kelchtermans, G. (2013). Sense-making and structure in teachers' reception of educational reform. A case study on statistics in the mathematics curriculum (vol 29, pg 13, 2013). Teaching and Teacher Education, 31(1), 67-67. https://doi.org/10.1016/j.tate.2012.08.004

Mayer, R. E. (1987). Educational psychology: A cognitive approach. Scott Foresman \& Company.

McCoy, L. P. (1996). Computer-based mathematics learning. Journal of Research on Computing in Education, 28(4), 438-460. https://doi.org/10.1080/08886504.1996.10782177 
Research, Society and Development, v. 10, n. 15, e235101522743, 2021

(CC BY 4.0) | ISSN 2525-3409 | DOI: http://dx.doi.org/10.33448/rsd-v10i15.22743

Pasqualotti, A., \& Freitas, C. M. D. S. (2002). MAT3D: a virtual reality modeling language environment for the teaching and learning of mathematics. CyberPsychology \& Behavior, 5(5), 409-422. https://doi.org/10.1089/109493102761022832

Santos, M. I., Breda, A., \& Almeida, A. M. (2017). Design approach of mathematics learning activities in a digital environment for children with autism spectrum disorders. Educational Technology Research and Development, 65(5), 1305-1323. https://doi.org/10.1007/s1 1423-017-9525-2

Silva, A. G. S., De Sousa, F. J. F., \& De Medeiros, J. L. (2020). O ensino da matemática: aspectos históricos. Research, Society and Development, 9(8), e488985850. http://dx.doi.org/10.33448/rsd-v9i8.5850

Slezáková, J. (2011). Geometrická představivost v rovině (Doctoral dissertation, disertační práce), PřF UP, Olomouc. https://theses.cz/id/op6350/?lang=en

Uygan, C., \& Kurtuluş, A. (2016). Effects of teaching activities via Google Sketchup and concrete models on spatial skills of preservice mathematics teachers. Turkish Journal of Computer and Mathematics Education (TURCOMAT), 7(3), 510-535. https://doi.org/10.16949/turkbilmat.273993

Van Hiele, P. M. (1986). Structure and insight: A theory of mathematics education. Academic press.

Watt, H. M. (2004). Development of adolescents' self-perceptions, values, and task perceptions according to gender and domain in 7th-through 11th-grade Australian students. Child development, 75(5), 1556-1574. https://doi.org/10.1111/j.1467-8624.2004.00757.x

Yilmaz, H. B. (2009). On the development and measurement of spatial ability. International Electronic Journal of Elementary Education, 1(2), 83-96. https://www.iejee.com/index.php/IEJEE/article/view/279/302 